\title{
A novel concept of domination in $m$-polar interval-valued fuzzy graph and its application
}

\author{
Sanchari Bera ${ }^{1}$ (D) Madhumangal Pal $^{1}$ (D)
}

Received: 10 January 2021 / Accepted: 31 July 2021 / Published online: 29 August 2021

(C) The Author(s), under exclusive licence to Springer-Verlag London Ltd., part of Springer Nature 2021

\begin{abstract}
The concept of domination is one of the most significant topics in graph theory to handle unpredictable phenomena. In this study, an unprecedented idea of domination is introduced in m-polar interval-valued fuzzy graph ( $m$-PIVFG). Domination number (DN), isolated vertex, total dominating set, independent set of domination on $m$-PIVFG are discussed. Some algebraic properties of domination on $m$-PIVFG are investigated. Weak domination, strong domination, split and non-split domination, cototal and global dominating sets on $m$-PIVFG are investigated with some fundamental hypotheses and models. We explore the concept of domination in $m$-PIVFG by solving a case study of locating new facilities to handle a catastrophe reaction activity due to the "COVID-19 pandemic" in West Bengal, India. Ultimately, conclusions and avenues of future scopes are placed at the end of this study.
\end{abstract}

Keywords $m$-PIVFG · Domination in $m$-PIVFG - Total dominating set · Independent dominating set on $m$-PIVFG . Split dominating set on $m$-PIVFG

$\begin{array}{ll}\text { Abbreviations } \\ \text { FS } & \text { Fuzzy set } \\ \text { FG } & \text { Fuzzy graph } \\ \text { DS } & \text { Dominating set } \\ \text { DN } & \text { Domination number } \\ \text { IVFS } & \text { Interval-valued fuzzy set } \\ \text { IVFG } & \text { Interval-valued fuzzy graph } \\ m \text {-PFS } & m \text {-polar fuzzy set } \\ m \text {-PFG } & m \text {-polar fuzzy graph } \\ m \text {-PIVFS } & m \text {-polar interval-valued fuzzy set } \\ m \text {-PIVFG } & m \text {-polar interval-valued fuzzy graph }\end{array}$

Madhumangal Pal

mmpalvu@mail.vidyasagar.ac.in; mmpalvu@gmail.com

Sanchari Bera

maths.sanchari@gmail.com

1 Department of Applied Mathematics with Oceanology and Computer Programming, Vidyasagar University, Midnapore 721 102, India

\section{Introduction}

Initially, graph theory plays an important role in various fields of mathematics as well as computer science such as group theory, topology, diagram representation, probability, numerical analysis, matrix theory and so on. Later day by day it has spread its branches in various fields of physics, biochemistry, biology, engineering field, operations research, astronomy, management science and most importantly in computer science. Graphs are the pictorial portrayal that connects the objects and features their data. To signify a real-world issue, this connection is most important. The fuzzy concept deals with haziness in operation research, human thinking, pattern recognition, expert systems, computer science, management science, control engineering, artificial intelligence, robotics, clustering analysis, and so on. Watching the immense utilization of graph theory, a numerical structure characterized as $G=(V, E)$ along with a great deal of vertices $V$ and edges $E$ was introduced. When there is a case of uncertainty and ambiguity in connection in either vertices or edges or both then the corresponding graph can be transferred to the fuzzy graph. 


\subsection{Motivation}

Fuzzy graph theory has wide range of applications in decision-making problems, facility location problems, covering problems. The fuzzy graph theory is tracking down an expanding number of uses for demonstrating continuous frameworks where the degree of data innate in the framework relies upon various levels of exactness. These days, scientists have two primary issues. From one perspective, people depend upon basic frameworks like a few basic infections, transportation, power, and sewage systems, water supply. Fuzzy graph can be used in different facility problems which are listed following:

- Health issue (Hospitals, ambulance)

- Electricity transmission and distribution

- Oil, gas and minerals production, transport and distribution

- Networking systems

- Water supply and drainage system

- Financial areas (Post office, Bank)

- Transport systems (Bus, rail, airports, ship).

Second issue is discovering optimal solution. It is found in numerous spaces of current science, innovation and financial aspects. After observing these two types of problems, location routing problems have been introduced in this paper. The world is now facing most dangerous COVID-19 pandemic situation. As, the decision making is the main piece of our life, which has a markable importance during this COVID-19 pandemic and it will profoundly influence our day by day life; so this paper is of deep significance to examine and make fundamental strategies to beat the present circumstance.

\subsection{Literature review}

The classical set theory introduced by Cantor cannot be dealing with this haziness and ambiguity. In 1736, Euler displayed the concept about Graph from the Konigsberg bridge problem. For taking care of different dynamic issues in real life, fuzzy set along with fuzzy relation have been applied. Based on Zadeh's conception on the fuzzy set, Kauffman [20] initiated the idea about the fuzzy graph. Later on, Rosenfeld [31] discussed this concept and developed definitions of various fuzzy notions like fuzzy vertex and edges, fuzzy paths, fuzzy cycles and so on which have been successfully implemented in medical diagnosis, engineering and manufacturing science etc. Afterwards, Bhattacharya [8] incorporated a few comments on fuzzy graphs in 1987. The opinion of a fuzzy set and bipolar fuzzy sets were extended by Zhang [41, 42] in 1994. Mordeson and Peng [23] explored the idea about complement on fuzzy graph and shown a couple of properties and operations on it. Shanon and Atnassov [34] incorporated the idea of intuitionistic fuzzy graphs and some properties on it. Afterwards, this concept was depicted by Sunitha and Kumar [37, 38]. Nagoorgani and Radha [26] presented a regular fuzzy graph to discover the density of fuzzy graph in 2008. Nagoorgani and Malarvizhu [25] studied on isomorphic properties and defined self-complementary fuzzy graphs. A deeper study on IVFG was done by Hongmei and Lianhua [18] in 2009 and by Akram and Dudek [1] in 2011. The ideas of bipolar fuzzy set and $m$-PFS were detailed by Chen et al. [9] in 2014. A couple of properties identified with isomorphism and supplement of IVFG were focused by Talebi and Rashmanlou [39]. Afterwards, Ghorai and Pal [14-17] examined several belongings on $m$-PFGs such as isomorphism, density and some other operations on it. Pramanik and pal [29] investigated different kinds of planar graphs. In fact, various extensions of fuzzy graphs are incorporated to handle the dubiousness of this present reality issue. Fuzzy graphs are used several models to tackle different dynamic issues in this uncertain environment. Various sorts of examination on summed up fuzzy graphs were handled by Samanta and Pal [32, 33]. Recently in 2019, radio fuzzy graphs were discussed by Mahapatra et al. [21]. Most recently in 2020, Jana et al. [19] studied on bipolar fuzzy dombi prioritized aggregation operators.

During the 1850s, an investigation of DSs in graph theory began simply as an issue in the round of chess. Chess devotees in Europe observed the issue of deciding the minimum number of queens that can be put on a chessboard with the goal that all the squares are either assaulted by a queen or involved by a queen. In 1962, the idea of domination in graphs was presented by Ore [27] and Berge [7], and thereafter concentrated by Cockayne and Hedetniemi $[10,11]$. Utilizing effective edges Somasundaram [35, 36] explored domination in fuzzy graphs. Afterwards, utilizing strong arc segments domination in the fuzzy graph was talked about by Nagoorgani and Chandrasekaran [24]. In the fuzzy graph, the concept of strong and weak domination was initiated by Gani and Ahamed [13]. The idea of 2-DS and secure DSs were investigated by Merouane and Chellali [22] in 2015. Motivating from different types of research on domination in fuzzy graphs [28], we focus on exploring domination in $m$-PIVFG. A more detailed classification of some related research is presented in Table 1 .

\subsection{Novelties}

The primary commitment of this investigation is as per the following: 
Table 1 Characteristics of some related research

\begin{tabular}{|c|c|c|c|c|c|c|}
\hline References & Year & FG & IVFG & $m$-PFG & Domination & $m$-PIVFG \\
\hline Ore [27] & 1962 & $x$ & $x$ & $\times$ & $\boldsymbol{\nu}$ & $\times$ \\
\hline Kauffman [20] & 1973 & レ & $\times$ & $\times$ & $\times$ & $\times$ \\
\hline Cockayne and Hedetniemi [10] & 1977 & $\times$ & $\times$ & $\times$ & レ & $\times$ \\
\hline Cockayne et al. [11] & 1980 & $x$ & $x$ & $\times$ & $\boldsymbol{V}$ & $\times$ \\
\hline Somasundaram and Somasundaram [36] & 1998 & レ & $\times$ & $\times$ & レ & $\times$ \\
\hline Nagoorgani and Chandrasekaran [24] & 2006 & レ & $x$ & $x$ & レ & $\times$ \\
\hline Gani and Ahamed [13] & 2007 & レ & $\times$ & $\times$ & レ & $\times$ \\
\hline Akram and Dudek [1] & 2011 & レ & レ & $\times$ & $\times$ & $\times$ \\
\hline Ghorai and Pal [14] & 2015 & $レ$ & $x$ & レ & $x$ & $\times$ \\
\hline Akram and Waseem [2] & 2016 & レ & $x$ & レ & $\times$ & $\times$ \\
\hline Ghorai and Pal [16] & 2017 & レ & $x$ & レ & $x$ & $\times$ \\
\hline Akram et al. [4] & 2017 & レ & レ & $x$ & レ & $\times$ \\
\hline Akram and Waseem [3] & 2017 & レ & レ & $x$ & レ & $\times$ \\
\hline Rashmanlou et al. [30] & 2018 & $レ$ & レ & $\times$ & $x$ & $\times$ \\
\hline Bera and Pal [6] & 2019 & レ & レ & レ & $\times$ & レ \\
\hline This study & 2020 & レ & レ & $\nu$ & レ & レ \\
\hline
\end{tabular}

- A novel conceptualization of domination on $m$-PIVFGs is introduced.

- Definitions of effective edges, size, degree, and isolated vertex in $m$-PIVFG with their examples are presented.

- Several types of domination on $m$-PIVFG are defined with suitable examples.

- Results on various kinds of domination of $m$-PIVFG are described.

- Real-life application examples of domination on $m$ PIVFG are discussed.

\subsection{Organization of the paper}

The remainder of the paper is sorted out as follows: The first section describes the historical backgrounds of graph theory. Our point and inspiration are additionally noted down in Section 1. Some preliminary basic ideas regarding FGs, $m$-PFGs, IVFGs with suitable examples are explained in Sect. 2. In Sect. 3, order, size, cardinality, and domination in $m$-PIVFG are discussed with examples. DN, isolated vertex, total DS, independent set of domination on $m$-PIVFG are also illustrated with some examples. Some important results related to domination on $m$-PIVFG are also discussed here. Definitions of different types of domination on $m$-PIVFG are investigated with supportive examples in Sect. 4. A few hypotheses and suggestions identified with these definitions are talked about with their comparing proofs. Section 5 incorporated a real-world problem regarding domination on $m$-PIVFG. Here we set up a disaster management problem with the algorithm.
Section 6 delineates a synopsis of this article alongside avenues of future scopes.

\section{Preliminaries}

In this piece of this paper, we quickly concentrate some essential phrasings of FS, FGs, $m$-PFS and $m$-PFGs which are utilized here. The essential meaning of IFGs is additionally illustrated.

In 1965, fuzzy sets, augmentation of the old style idea of the set were suggested by Zadeh [40]. We recall the definitions of $m$-PFG and IVFG from [5]. For more clarification, one can visit that manuscript thoroughly. All through this article, we utilize the documentation $G^{*}$ as an old style classical graph and $G=(V, A, B)$ as an $m$-PIVFG.

Definition 1 [6] An $m$-PIVFG $G=(V, A, B)$ consists of a non-empty set $V$ with two IVFSs (i) $A: V \rightarrow[0,1]^{m}$ an $m$ FS in $V$ and (ii) $B: V \times V \rightarrow[0,1]^{m}$ an $m$-PFR on $V$ and $\mu: V \times V \rightarrow[0,1]^{m}$ with underlying conditions $p_{i} \circ$ $\mu_{A}(x)=\left[p_{i} \circ \mu_{A}^{l}(x), p_{i} \circ \mu_{A}^{u}(x)\right], 0 \leq \mu_{A}^{l}(x) \leq \mu_{A}^{u}(x) \leq 1$ and $p_{i} \circ \mu_{B}(x y)=\left[p_{i} \circ \mu_{B}^{l}(x y), p_{i} \circ \mu_{B}^{u}(x y)\right]$,

$0 \leq \mu_{B}^{l}(x y) \leq \mu_{B}^{u}(x y) \leq 1$ and for every $i=1,2, \ldots, m$, satisfying $\quad p_{i} \circ \mu_{B}^{l}(x y) \leq \min \left\{p_{i} \circ \mu_{A}^{l}(x), p_{i} \circ \mu_{A}^{l}(y)\right\} \quad, \quad p_{i} \circ$ $\mu_{B}^{u}(x y) \leq \min \left\{p_{i} \circ \mu_{A}^{u}(x), p_{i} \circ \mu_{A}^{u}(y)\right\}, \forall x, y \in V$.

Definition 2 [6] The complement $\bar{G}=(V, \bar{A}, \bar{B})$ of $G$ is also an $m$-PIVFG where $p_{i} \circ \mu_{\bar{B}}(x y)=\left[p_{i} \circ \mu_{\bar{B}}^{l}(x y), p_{i} \circ\right.$ $\left.\mu_{\bar{B}}^{u}(x y)\right], \quad p_{i} \circ \mu_{\bar{B}}^{l}(x y)=\min \left\{p_{i} \circ \mu_{A}^{l}(x), p_{i} \circ \mu_{A}^{l}(y)\right\}-\quad p_{i} \circ$ 
$\mu_{B}^{l}(x y), \quad$ and $\quad p_{i} \circ \mu_{\bar{B}}^{u}(x y)=\min \left\{p_{i} \circ \mu_{A}^{u}(x), p_{i} \circ \mu_{A}^{u}(y)\right\}-$ $p_{i} \circ \mu_{B}^{u}(x y)$ for every $i$ and $x, y \in V$.

Definition 3 [36] A dominating set $S$ in $G=(V, E)$ is defined as for every $x \in V-S$ dominates some $y \in S$, where $S \subseteq V$.

In the next section, domination on $m$-PIVFG is defined with suitable examples. Some basic terminologies related to domination are also explained.

\section{Domination in $m$-PIVFG}

Herein, the concept of domination in $m$-PIVFG is explored with examples. In this section, various terms related to domination on $m$-PIVFG are also discussed. Also, in this part we described minimal domination, maximal domination and total domination in $m$-PIVFG with suitable examples. Some significant outcomes are likewise clarified with their proofs.

Definition 4 An edge $B(x y)$ for an $m$-PIVFG $G=$ $(V, A, B) \quad$ is effective if $p_{i} \circ \mu_{B}^{l}(x y)=p_{i} \circ$ $\min \left\{\mu_{A}^{l}(x), \mu_{A}^{l}(y)\right\}, p_{i} \circ \mu_{B}^{u}(x y)=p_{i} \circ \min \left\{\mu_{A}^{u}(x), \mu_{A}^{u}(y)\right\}$, for $x, y \in V$.

Definition 5 The lower sum of the membership values of all effective edges incident at $x$ of an $m$-PIVFG $G=$ $(V, A, B)$ is lower effective degree of $x$ and is represented by $d_{B}^{l}(x)$ and is given by $d_{B}^{l}(x)=\sum_{i} p_{i} \circ \mu_{A}^{l}(x)$.

Definition 6 The upper sum of the membership values of all effective edges incident at $x$ of $G=(V, A, B)$ is upper effective degree of $x$ and is represented by $d_{B}^{u}(x)$ and is given by $d_{B}^{u}(x)=\sum_{i} p_{i} \circ \mu_{A}^{u}(x)$.

Definition 7 The order $\tilde{p}$ and size $\tilde{q}$ of $G=(V, A, B)$ is defined as $\tilde{p}=\sum_{x \in V} \frac{1+\sum_{i} p_{i} \circ \mu_{A}^{l}(x)+\sum_{i} p_{i} \circ \mu_{A}^{u}(x)}{2}$ and $\tilde{q}=$ $\sum_{x y \in E} \frac{1+\sum_{i} p_{i} \circ \mu_{B}^{l}(x y)+\sum_{i} p_{i} \circ \mu_{B}^{u}(x y)}{2}, \forall x \in V$ and $\forall x y \in E$.

Definition 8 Let $\tilde{S} \subseteq V$. Then cardinality of $\tilde{S}$, $\mathcal{N}(\tilde{S})=\sum_{x \in \tilde{S}} \frac{1+\sum_{i} \mu_{A}^{u}(x)-\sum_{i} \mu_{A}^{l}(x)}{2}$.

Definition 9 Two vertices $x$ and $y$ are neighbors to each other in an $m$-PIVFG if for each $i$, for $x, y \in V$, either one of the accompanying hold

i. $\quad p_{i} \circ \mu_{B}^{l}(x y)>0, p_{i} \circ \mu_{B}^{u}(x y)>0$.

ii. $p_{i} \circ \mu_{B}^{l}(x y)=0, p_{i} \circ \mu_{B}^{u}(x y)>0$.

iii. $p_{i} \circ \mu_{B}^{l}(x y)>0, p_{i} \circ \mu_{B}^{u}(x y)=0$.

Definition 10 We define $x$ dominates $y$ for an $m$-PIVFG $G=(V, A, B) \quad$ if for $\quad x, y \in V, \quad p_{i} \circ \mu_{B}^{l}(x y)=\min \left\{p_{i} \circ\right.$
$\left.\mu_{A}^{l}(x), p_{i} \circ \mu_{A}^{l}(y)\right\}, \quad p_{i} \circ \mu_{B}^{u}(x y)=\min \left\{p_{i} \circ \mu_{A}^{u}(x), p_{i} \circ\right.$ $\left.\mu_{A}^{u}(y)\right\}$, for $i=1,2, \ldots, m$. Let $\tilde{S} \subseteq V$. $\tilde{S}$ is a DS in $G=$ $(V, A, B)$ if for every $y \in V-S, \exists x \in S$ so that $x$ dominates $y$.

The domination number $\Upsilon(G)$ means the minimum cardinality of all DS in $G$ and $\Upsilon(G)=\min _{\tilde{S} \in V} \sum_{x \in \tilde{S}} \frac{1+\sum_{i} \mu_{A}^{u}(x)-\sum_{i} \mu_{A}^{l}(x)}{2}$.

Example 1 Let $G=(V, A, B)$ be an $m$-PIVFG (Fig. 1), where

$$
\begin{aligned}
A= & \frac{a}{\langle[0.1,0.5],[0.4,0.7],[0.3,0.8]\rangle}, \frac{b}{\langle[0.2,0.6],[0.4,0.5],[0.2,0.5]\rangle}, \\
& \frac{c}{\langle[0.1,0.3],[0.6,0.7],[0.3,0.5]\rangle}, \frac{d}{\langle[0.1,0.5],[0.4,0.7],[0.3,0.8]\rangle}, \\
& \left.\frac{e}{\langle[0.0,0.4],[0.5,0.8],[0.2,0.6]\rangle}\right) . \\
B= & \left(\frac{a b}{\langle[0.1,0.5],[0.4,0.5],[0.2,0.5]\rangle}, \frac{b c}{\langle[0.1,0.3],[0.6,0.7],[0.3,0.5]\rangle},\right. \\
& \frac{d d}{\langle[0.1,0.3],[0.4,0.7],[0.3,0.5]\rangle}, \frac{d e}{\langle[0.0,0.3],[0.3,0.6],[0.1,0.5]\rangle}, \\
& \frac{e a}{\langle[0.0,0.4],[0.4,0.7],[0.2,0.6]\rangle}, \frac{b e}{\langle[0.0,0.4],[0.4,0.5],[0.2,0.5]\rangle} \\
& \left.\frac{b d}{\langle[0.1,0.5],[0.4,0.5],[0.2,0.5]\rangle}\right) .
\end{aligned}
$$

Here, DSs are $\{b, c\},\{b\},\{a, b\},\{a, c\},\{a, b, c\}$.

Definition 11 A minimal DS $\tilde{S}$ of an $m$-PIVFG is a DS where there does not exist any DS which is proper subset of $\tilde{S}$.

Definition 12 Lower and upper DN of an $m$-PIVFG is denoted by $\tilde{d}(G)$ and $\tilde{D}(G)$, respectively, and defined by minimum cardinality and maximum cardinality of all minimal DS of that $m$-PIVFG, respectively.

Example 2 From Example 1, Fig. 1, minimal DS is $\{b\}$.

\section{Note 1}

1. For $G=(V, A, B), \quad x$ dominates $y \Rightarrow y$ dominates $x$. That means domination is a symmetric relation.

2. If $p_{i} \circ \mu_{B}^{l}(x y)<\min \left\{p_{i} \circ \mu_{A}^{l}(x), p_{i} \circ \mu_{A}^{l}(y)\right\}$ and $p_{i} \circ$ $\mu_{B}^{u}(x y)<\min \left\{p_{i} \circ \mu_{A}^{u}(x), p_{i} \circ \mu_{A}^{u}(y)\right\}, \quad \forall x, y \in V \quad$ and for $i=1,2, \ldots, m$, then $V$ is the only DS in an $m$ PIVFG $G=(V, A, B)$.

Definition 13 For $G=(V, A, B)$, a vertex $x$ is an isolated vertex if for each $i, \quad p_{i} \circ \mu_{B}^{l}(x y)<\min \left\{p_{i} \circ \mu_{A}^{l}(x), p_{i} \circ\right.$ $\left.\mu_{A}^{l}(y)\right\}$ and $p_{i} \circ \mu_{B}^{u}(x y)<\min \left\{p_{i} \circ \mu_{A}^{u}(x), p_{i} \circ \mu_{A}^{u}(y)\right\} \forall v \in$ $V-\{x\}$ so that $N(x)=\phi$, i.e., there is an edge between $x \& y$. That means an isolated vertex does not dominate any other vertex in $G$. 
Fig. 1 DS in a 3-PIVFG $G$

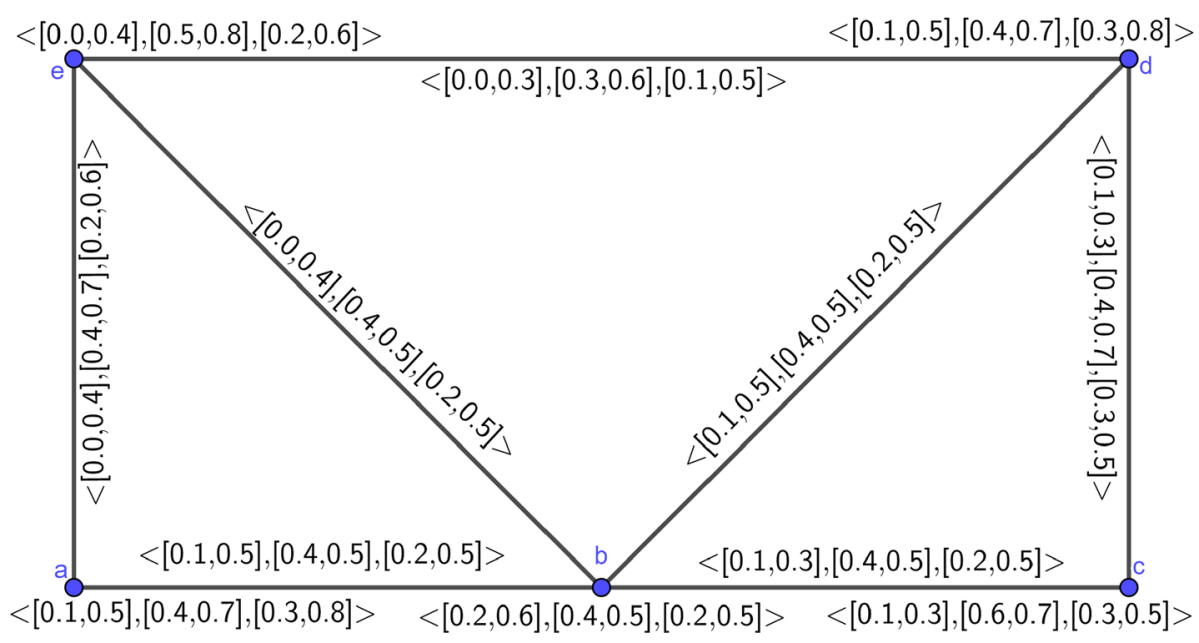

Example 3 Let us assume an $m$-PIVFG $G=(V, A, B)$ (Fig. 2), where

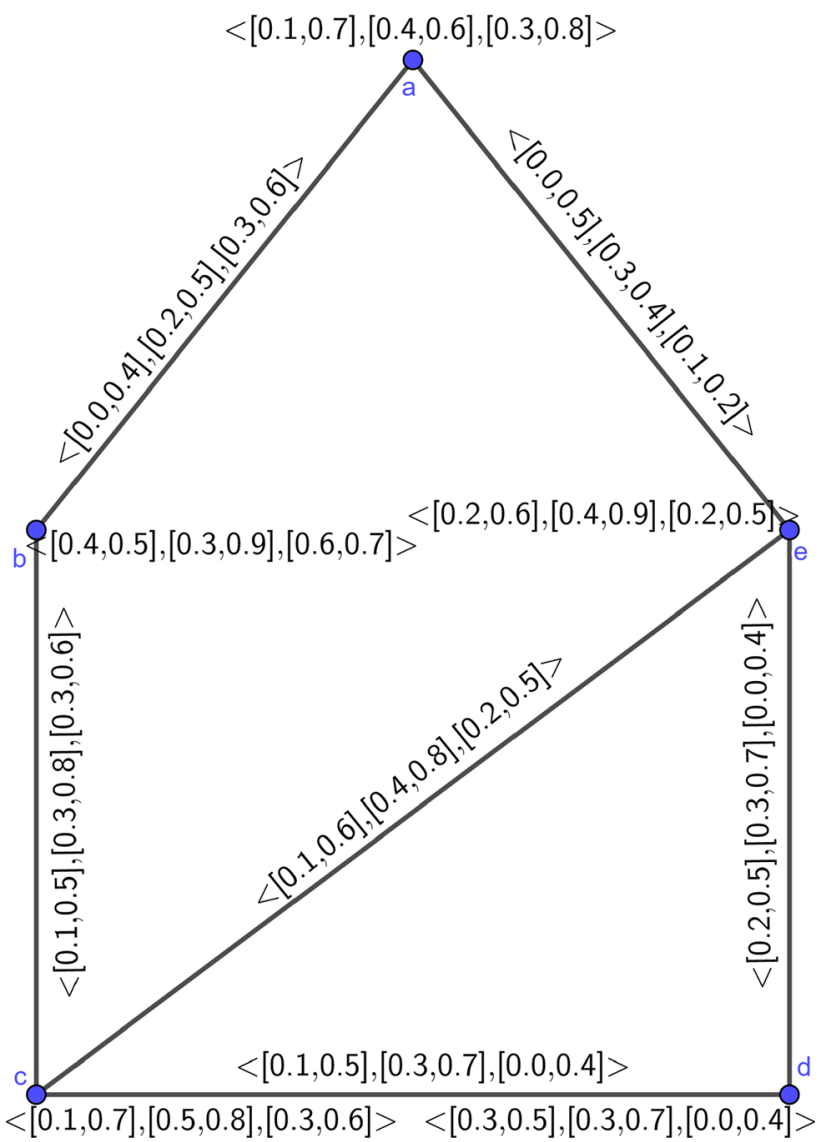

Fig. 2 Isolated vertex of an 3-PIVFG $G$

$$
\begin{aligned}
A= & \left(\frac{a}{\langle[0.1,0.7],[0.4,0.6],[0.3,0.8]\rangle}, \frac{b}{[0.4,0.5],[0.3,0.9],[0.6,0.7]},\right. \\
& \frac{c}{[0.1,0.7],[0.5,0.8],[0.3,0.6]} \\
& \left.\frac{d}{\langle[0.3,0.5],[0.3,0.7],[0.0,0.4]\rangle}, \frac{e}{\langle[0.2,0.6],[0.4,0.9],[0.2,0.5]\rangle}\right), \\
B= & \left(\frac{a b}{\langle[0.0,0.4],[0.2,0.5],[0.3,0.6]\rangle}, \frac{b c}{\langle[0.1,0.5],[0.3,0.8],[0.3,0.6]\rangle},\right. \\
& \frac{c d}{\langle[0.1,0.5],[0.3,0.7],[0.0,0.4]\rangle} \\
& \frac{d e}{\langle[0.2,0.5],[0.3,0.7],[0.0,0.4]\rangle}, \frac{e c}{\langle[0.0,0.5],[0.3,0.4],[0.1,0.2]\rangle}, \\
& \left.\frac{e c}{\langle[0.1,0.6],[0.4,0.8],[0.2,0.5]\rangle}\right) .
\end{aligned}
$$

Here, ' $a$ ' is an isolated vertex as $p_{i} \circ \mu_{B}^{l}(a b)<\min \left\{p_{i} \circ\right.$ $\left.\mu_{A}^{l}(a), p_{i} \circ \mu_{A}^{l}(b)\right\}$ and $p_{i} \circ \mu_{B}^{u}(a b)<\min \left\{p_{i} \circ \mu_{A}^{u}(a), p_{i} \circ\right.$ $\left.\mu_{A}^{u}(b)\right\}$ and also $p_{i} \circ \mu_{B}^{l}(a c)<\min \left\{p_{i} \circ \mu_{A}^{l}(a), p_{i} \circ \mu_{A}^{l}(c)\right\}$ and $p_{i} \circ \mu_{B}^{u}(a c)<\min \left\{p_{i} \circ \mu_{A}^{u}(a), p_{i} \circ \mu_{A}^{u}(c)\right\}$. Therefore, ' $a$ ' does not dominate any other vertex in $G$.

Definition 14 Let us suppose $G=(V, A, B)$ has no isolated vertices. A set $\tilde{D}$ from $V$ be a total DS on $G=$ $(V, A, B)$ if for each vertex $y \in V, \exists x \in \tilde{D}, x \neq y$ so that $x$ dominates $y$.

Definition 15 Lower (upper) total DN $\tilde{t}(G)(\tilde{T}(G))$ is the minimum (maximum) cardinality of a total DS on an $m$ PIVFG $G=(V, A, B)$.

Example 4 From Example 1, Fig. 1, minimal DS is $\{b\}$ and $\mathcal{N}\{a, c\}=1.85, \mathcal{N}\{a, b, c\}=2.75, \mathcal{N}\{b, c\}=1.65$, $\mathcal{N}\{a, b\}=2.0, \quad \mathcal{N}\{b\}=0.9 . \quad$ Hence,$\quad \tilde{t}(G)=0.9$ $\tilde{T}(G)=2.75$

Theorem 1 For an $m$-PIVFG $G=(V, A, B), \tilde{t}(G)+$ $\overline{\tilde{t}(G)} \leq 2 \tilde{p}$, where $\tilde{t}(G)$ and $\tilde{\tilde{t}(G)}$ are total $D N$ of $G$ and its complement $\bar{G}$, respectively, and the equality holds iff 
$0<p_{i} \circ \mu_{B}^{l}(x y)<\min \left\{p_{i} \circ \mu_{A}^{l}(x), p_{i} \circ \mu_{A}^{l}(y)\right\}$ and $0<p_{i} \circ$ $\mu_{B}^{u}(x y)<\min \left\{p_{i} \circ \mu_{A}^{u}(x), p_{i} \circ \mu_{A}^{u}(y)\right\} \forall x, y \in V$.

Proof $\tilde{t}(G)=\tilde{p} \quad$ iff $\quad p_{i} \circ \mu_{B}^{l}(x y)=\min \left\{p_{i} \circ \mu_{A}^{l}(x), p_{i} \circ\right.$ $\left.\mu_{A}^{l}(y)\right\}$ and $p_{i} \circ \mu_{B}^{u}(x y)=\min \left\{p_{i} \circ \mu_{A}^{u}(x), p_{i} \circ \mu_{A}^{u}(y)\right\}$ for all $x, y \in V$. Again since $\overline{\tilde{t}(G)}=\tilde{p}$ iff $p_{i} \circ \mu_{\bar{B}}^{l}(x y)=$ $\min \left\{p_{i} \circ \mu_{A}^{l}(x), p_{i} \circ \mu_{A}^{l}(y)\right\}-p_{i} \circ \mu_{B}^{l}(x y)$ and $p_{i} \circ \mu_{\bar{B}}^{u}(x y)=$ $\min \left\{p_{i} \circ \mu_{A}^{u}(x), p_{i} \circ \mu_{A}^{u}(y)\right\}-p_{i} \circ \mu_{B}^{u}(x y)$ for all $x, y \in V$, these two results conclude that $p_{i} \circ \mu_{B}^{l}(x y)>0 \& p_{i} \circ$ $\mu_{B}^{u}(x y)>0$. Therefore, $\tilde{t}(G)+\overline{\tilde{t}(G)}=2 \tilde{p} \quad$ iff $\quad 0<p_{i}$ 。 $\mu_{B}^{l}(x y)<\min \left\{p_{i} \circ \mu_{A}^{l}(x), p_{i} \circ \mu_{A}^{l}(y)\right\} \quad$ and $\quad 0<p_{i} \circ$ $\mu_{B}^{u}(x y)<\min \left\{p_{i} \circ \mu_{A}^{u}(x), p_{i} \circ \mu_{A}^{u}(y)\right\}$ for all $x, y \in V$.

Theorem 2 Iff one of the below mentioned criteria holds then a DS D be minimal.

i. $N(x) \cap \tilde{D}=\phi$

ii. There is a vertex $y \in V-\tilde{D}$ such that $N(y) \cap \tilde{D}=x$, for each $x \in \tilde{D}$.

Proof For a minimal DS $\tilde{D}$ of an $m$-PIVFG $G=(V, A, B)$, for every vertex $x \in \tilde{D}$, no proper subset of $\tilde{D}$ that is $\tilde{D_{1}}=$ $\tilde{D}-x$ is DS. Therefore, any vertex in $\tilde{D_{1}}$ cannot dominate any vertex $y \in V-\tilde{D_{1}}$. If $y=x$, then $x$ cannot dominate any vertex in $\tilde{D_{1}}$. If $y \neq x$, then $y$ is not dominated by $\tilde{D_{1}}$ but is dominated by $\tilde{D}$. Thus $y$ can dominate only $x$ in $\tilde{D}$ that means $N(y) \cap \tilde{D}=x$.

Conversely, let $\tilde{D}$ holds one of the two given criterias. If $\tilde{D}$ is not a minimal DS, then $\exists x \in \tilde{D}$ so that $\tilde{D_{1}}=\tilde{D}-\{x\}$ is dominating. That means atleast one vertex in $\tilde{D_{1}}$ can dominate $x$ hence (i) does not hold. Again if $\tilde{D_{1}}$ is a DS, then atleast one vertex in $\tilde{D_{1}}$ can dominate every vertex in $V-\tilde{D}$ that implies (ii) also does not hold, which contradict our assumption. $\therefore \tilde{D}$ must be a minimal DS.

Definition 16 Let $G=(V, A, B)$ be an $m$-PIVFG. A set $I \subseteq V$ is called an independent set of $G$ if $p_{i} \circ$ $\mu_{B}^{l}(x y)<\min \left\{p_{i} \circ \mu_{A}^{l}(x), p_{i} \circ \mu_{A}^{l}(y)\right\}, \quad p_{i} \circ \mu_{B}^{u}(x y)<\min$ $\left\{p_{i} \circ \mu_{A}^{u}(x), p_{i} \circ \mu_{A}^{u}(y)\right\}$ for all $x, y \in I$ and for each $i$.

Definition 17 An independent set $I$ of an $m$-PIVFG $G=$ $(V, A, B)$ is called maximal independent set of $G$ if for every vertex $x \in V-I$, the set $I \cup\{x\}$ is not independent.

Definition 18 For an $m$-PIVFG $G=(V, A, B)$, the lower and upper independence number mean the minimum cardinality and maximum cardinality among all maximal independent sets, respectively, and represented by $\tilde{i}(G)$ and $\tilde{I}(G)$, respectively.
Definition 19 The upper independence number of an $m$ PIVFG $G=(V, A, B)$ denoted by is defined as the maximum cardinality among all maximal independent sets of $G$.

Example 5 Let us consider $G=(V, A, B)$ (Fig. 3), where

$$
\begin{aligned}
A= & \frac{a}{\langle[0.4,0.6],[0.3,0.9],[0.1,0.7]\rangle}, \frac{c}{\langle[0.3,0.7],[0.4,0.8],[0.2,0.7]\rangle}, \\
& \frac{c}{\langle[0.2,0.6],[0.4,0.7],[0.1,0.5]\rangle}, \\
& \left.\frac{d}{\langle[0.5,0.8],[0.6,0.9],[0.2,0.4]\rangle}, \frac{a}{\langle\langle 0.2,0.7],[0.4,0.5],[0.1,0.6]\rangle}\right) . \\
B= & \left(\frac{a b}{\langle[0.2,0.5],[0.2,0.6],[0.0,0.5]\rangle}, \frac{a c}{\langle[0.1,0.4],[0.2,0.6],[0.0,0.3]\rangle},\right. \\
& \frac{a d}{\langle[0.3,0.5],[0.2,0.8],[0.0,0.2]\rangle}, \\
& \frac{a e}{\langle[0.2,0.5],[0.3,0.7],[0.0,0.4]\rangle}, \frac{b c}{\langle[0.0,0.5],[0.3,0.4],[0.1,0.2]\rangle}, \\
& \frac{b d}{\langle[0.1,0.6],[0.4,0.8],[0.2,0.5]\rangle} \\
& \frac{b e}{\langle[0.2,0.5],[0.3,0.7],[0.0,0.4]\rangle}, \frac{c e}{\langle[0.1,0.4],[0.2,0.5],[0.0,0.2]\rangle}, \\
& \frac{c e}{\langle[0.1,0.6],[0.4,0.8],[0.2,0.5]\rangle} \\
& \left.\frac{d e}{\langle[0.2,0.5],[0.3,0.7],[0.0,0.4]\rangle}\right)
\end{aligned}
$$

Here, independent sets are $M_{1}=\{a, d\}, M_{2}=$ $\{a, c\}, M_{3}=\{a, b\}, M_{4}=\{c, d\}, M_{5}=\{a, c, d\}$. Maximal independent sets are $M_{3}=\{a, b\}, M_{5}=\{a, c, d\}$. Thus, $\mathcal{N}\left(M_{3}\right)=2.35$ and $\mathcal{N}\left(M_{5}\right)=3.15$. Therefore, $\tilde{i}(G)=$ 2.35 and $\tilde{I}(G)=3.15$.

\section{Proposition 1 For any $m-P I V F G, \Upsilon(G) \leq \tilde{i}(G)$.}

Example 6 By direct calculation from (Fig. 3), the set $\{e\}$ is a minimal DS and maximal independent sets are $\{a, c, d\}$ and $\{a, b\} . \mathcal{N}\{e\}=1.05$. Hence, $\Upsilon(G)=1.05$ Again, $\tilde{i(} G)=2.35$. Thus clearly, $\Upsilon(G) \leq \tilde{i}(G)$.

Corollary 1 For a complete $m$-PIVFG $G=(V, A, B)$, $\Upsilon(G)<\tilde{i}(G)$.

Theorem 3 A set $I \subseteq V$ is a maximal independent set of an $m-P I V F G G=(V, A, B)$ iff it is independent and $D S$ of $G$.

Proof let us consider a maximal independent set $I$. Then from the definition, for every $x \in V-I$, the set $I \cup\{x\}$ is not independent. Hence, for every vertex $x \in V-I, \exists y \in I$ so that $y$ can dominate $x$ which implies $I$ is a DS. $\therefore I$ is both independent and dominating. Conversely if we suppose that $I$ is not maximal independent set then $\exists x \in V-I$ such that $I \cup\{x\}$ is independent. Thus $x$ cannot dominate an $y$ in I. That implies, $I$ is not a DS, which contradict our assumption. $\therefore I$ is a maximal independent set.

Theorem 4 Every maximal independent set of an $m$ $P I V F G G=(V, A, B)$ is a minimal $D S$. 
Fig. 3 Independent sets of 3-PIVFG $G$

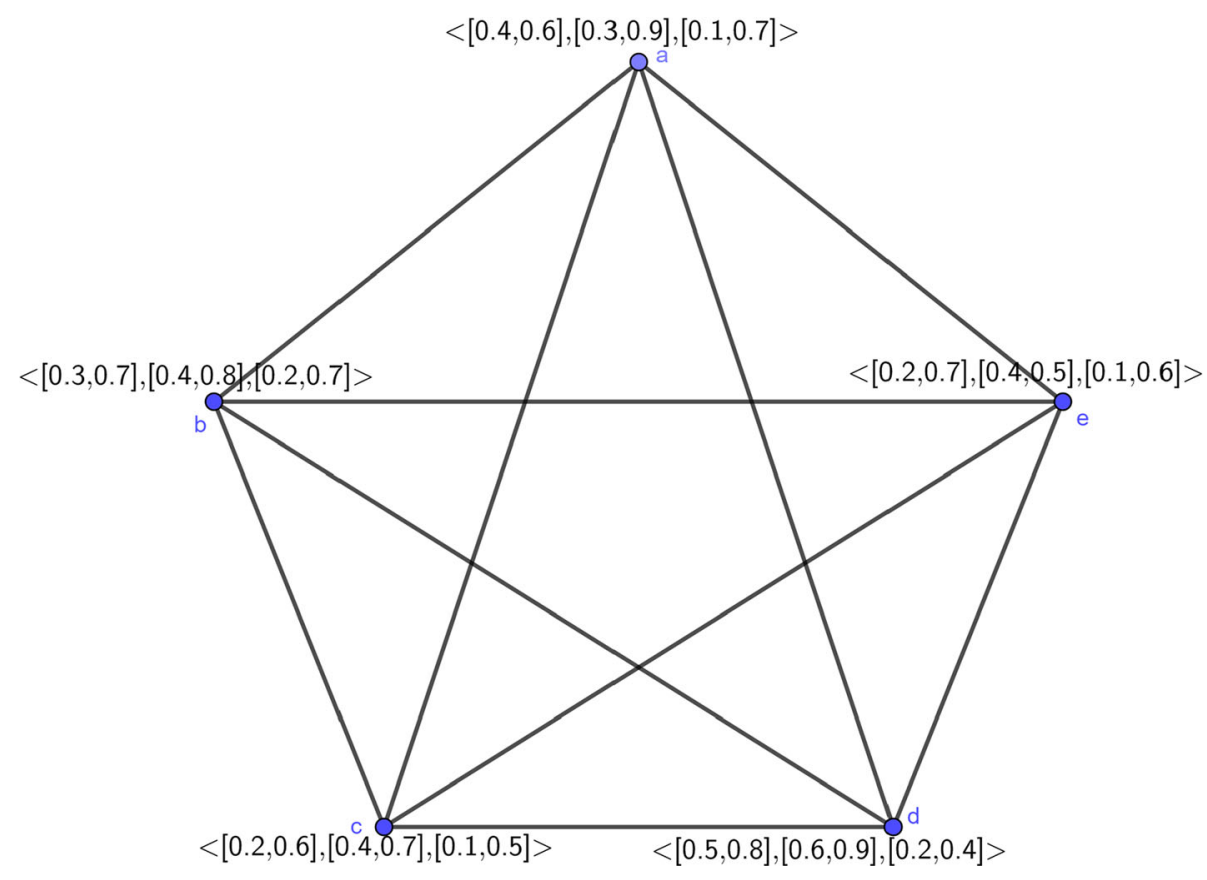

Proof For a maximal independent set $I$ of $G=(V, A, B), I$ is DS. If we consider $I$ being not minimal DS of $G$, then for atleast one vertex $x \in I$ so that $I-\{x\}$ is dominating that means $I-\{x\}$ dominates $V-(I-\{x\})$. Thus, there exists atleast one vertex in $I$ which dominates $x$. This contradict our assumption. $\therefore I$ is a minimal DS of $G=(V, A, B)$.

\section{Other dominations in $\boldsymbol{m}$-PIVFG}

Herein, various types of domination in $m$-PIVFG with reasonable models are characterized and a few hypotheses identified with that are portrayed.

\subsection{Strong and weak dominations in $m$-PIVFG}

Definition 20 For $x, y \in V$ of an $m$-PIVFG $G=(V, A, B)$, $x$ strongly dominates $y$ if $p_{i} \circ \mu_{B}^{l}(x y)=\min \left\{p_{i} \circ \mu_{A}^{l}(x), p_{i} \circ\right.$ $\left.\mu_{A}^{l}(y)\right\}, \quad p_{i} \circ \mu_{B}^{u}(x y)=\min \left\{p_{i} \circ \mu_{A}^{u}(x), p_{i} \circ \mu_{A}^{u}(y)\right\} \quad$ and $d_{B}^{u}(x) \geq d_{B}^{u}(y)$ and $d_{B}^{l}(x) \geq d_{B}^{l}(y)$ for $1 \leqslant i \leqslant m$. Similarly, $x$ weakly dominates $y$ if $p_{i} \circ \mu_{B}^{l}(x y)=\min \left\{p_{i} \circ \mu_{A}^{l}(x), p_{i} \circ\right.$ $\left.\mu_{A}^{l}(y)\right\}, \quad p_{i} \circ \mu_{B}^{u}(x y)=\min \left\{p_{i} \circ \mu_{A}^{u}(x), p_{i} \circ \mu_{A}^{u}(y)\right\} \quad$ and $d_{B}^{u}(x) \leq d_{B}^{u}(y)$ and $d_{B}^{l}(x) \leq d_{B}^{l}(y)$, for $1 \leqslant i \leqslant m$.

Definition 21 A set $\tilde{D} \subseteq V$ is a strong DS of an $m$-PIVFG $G$ if for every $y \notin \tilde{D}, \exists x \in \tilde{D}$ so that $x$ strongly dominates $y$.

Definition 22 A set $\tilde{D} \subseteq V$ is a weak DS of an $m$-PIVFG $G$ if for $y \notin \tilde{D}, \exists x \in \tilde{D}$ such that $x$ weakly dominates $y$.
Definition 23 Strong DN $\Upsilon_{s}(G)$ of an $m$-PIVFG $G$ is the minimum cardinality of all strong DS of an $m$-PIVFG $G$.

Weak DN $\Upsilon_{w}(G)$ of $G$ is the minimum cardinality of all weak DS of an $m$-PIVFG $G$.

Example 7 Consider a 3-PIVFG $G=(V, A, B)$ (shown in Fig. 4), where

$$
\begin{aligned}
A= & \left(\frac{a}{\langle[0.1,0.7],[0.4,0.6],[0.3,0.8]\rangle}, \frac{b}{\langle[0.4,0.5],[0.3,0.9],[0.6,0.7]\rangle},\right. \\
& \frac{c}{\langle[0.1,0.7],[0.5,0.8],[0.3,0.6]\rangle}, \\
& \left.\frac{d}{\langle[0.3,0.5],[0.3,0.7],[0.0,0.4]\rangle}\right) . \\
B= & \left(\frac{a b}{\langle[0.0,0.4],[0.2,0.5],[0.3,0.6]\rangle}, \frac{b c}{\langle[0.1,0.5],[0.3,0.8],[0.3,0.6]\rangle},\right. \\
& \frac{c d}{\langle[0.1,0.5],[0.3,0.7],[0.0,0.4]\rangle}, \\
& \left.\frac{d a}{\langle[0.2,0.5],[0.3,0.7],[0.0,0.4]\rangle}\right)
\end{aligned}
$$

For the edge $a b$, ' $b$ ' weakly dominates ' $a$ ' or we can say ' $a$ ' strongly dominates ' $b$ ' as $d_{B}^{u}(a)>d_{B}^{u}(b)$ and $d_{B}^{l}(a)>d_{B}^{l}(b)$, similarly ' $c$ ' strongly dominates ' $d$.' Here we consider the DS $\{a, c\}$. By calculation, we find it to be a strong DS. Hence, $\Upsilon_{s}(G)=2.2$.

\subsection{Split domination in $m$-PIVFG}

Theorem 5 For a complete $m$-PIVFG, $\Upsilon_{w}(G) \leqslant \Upsilon_{s}(G)$.

Definition 24 A DS $\tilde{D}$ of $G=(V, A, B)$ is split DS if the induced subgraph $\langle V-\tilde{D}\rangle$ of $G$ be disconnected. The split 


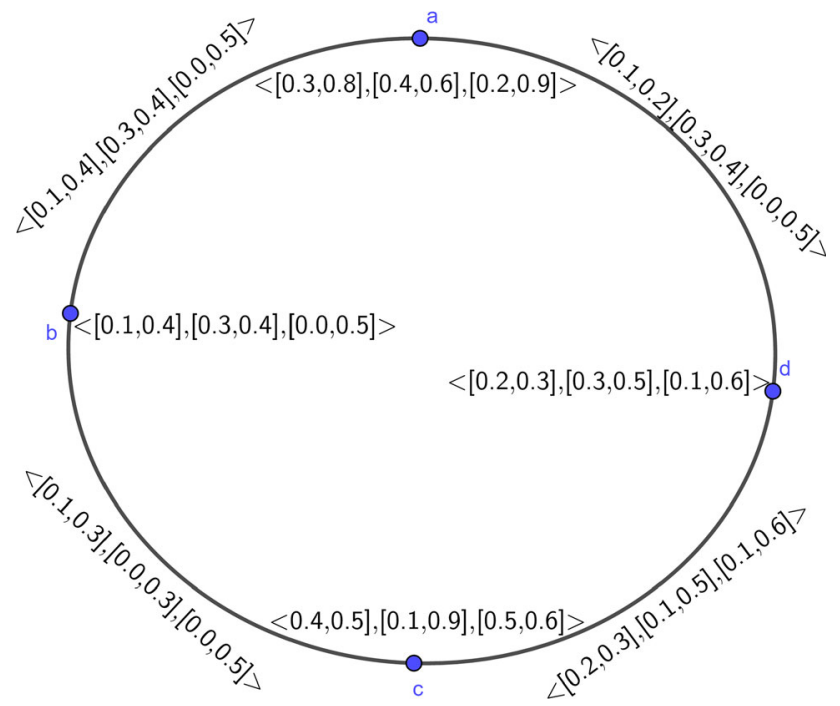

Fig. 4 Strong DS on a 3-PIVFG $G$

DN $\Upsilon_{\text {sp }}$ be the minimum cardinality of all split DS of that $m$-PIVFG.

Example 8 From (Fig. 4), Example 7, we consider a DS $\{a, c\}$. Thus $\langle V-\tilde{D}\rangle=\{b, d\}$ is disconnected. Therefore, $\{a, c\}$ is split DS on this 3-PIVFG $G=(V, A, B)$.

Corollary 2 Split DS exists if the graph is not complete and either complete contains a non-complete component or at least two non-trivial components.

Corollary 3 Every split $D S$ in an $m$-PIVFG is DS, i.e., $\Upsilon(G) \leqslant \Upsilon_{\mathrm{sp}}(G)$.

Theorem 6 A split DS D̃ of an $m$-PIVFG $G$ is minimal for each vertex $x \in \tilde{D}$, one of the accompanying holds:

i. $\exists y \in V-\tilde{D}$ so that $N(y) \cap \tilde{D}=\{x\}$.

ii. $x \in \tilde{D}$ be isolated.

iii. $\langle V-\tilde{D} \cup\{x\}\rangle$ being connected.

Proof Suppose for an minimal split DS $\tilde{D}$ of an $m$-PIVFG $\exists x \in \tilde{D}$ so that $X$ doesn't satisfy any of the above-mentioned conditions. Thereafter from i. \& ii., $\tilde{D_{1}}=\tilde{D}-\{x\}$ be DS. Again from the condition iii. $\langle V-\tilde{D}\rangle$ being disconnected implies $\tilde{D_{1}}$ as a split DS, which is a contradiction.

Definition 25 A strong split DS $\tilde{D}$ of $G=(V, A, B)$ is a DS where the induced subgraph $\langle V-\tilde{D}\rangle$ is totally disconnected with at least two vertices. The split DN $\Upsilon_{\text {ssp }}$ means the minimum cardinality of all strong split DS.

Example 9 From Example 7, (Fig. 4) we consider a DS $\{a, c\}$ where the induced subgraph $\langle V-\tilde{D}\rangle=\{b, d\}$ being disconnected with two vertices. Therefore, $\{a, c\}$ is strong split DS on this 3-PIVFG.

Theorem 7 A DS $\tilde{D}$ of $G=(V, A, B)$ be strong split DS iff the followings are satisfied.

i. $\quad V-\tilde{D}$ has at least two vertices.

ii. For any $x, y \in V-\tilde{D}$, every $x-y$ path consists at least a vertex of $\tilde{D}$.

Proof Proof of this theorem is straight forward.

Theorem 8 A strong split $D S$ of $G=(V, A, B)$ be minimal iff for each vertex $v \in \tilde{D}$, any of the accompanying condition is fulfilled.

i. $\exists x \in V-\tilde{D}$ so that $x$ is adjacent to $y \in \tilde{D}$.

ii. $y \in \tilde{D}$ be an isolated vertex.

\subsection{Non-split domination in $\boldsymbol{m}$-PIVFG}

Definition 26 A DS $\tilde{D}$ of $G=(V, A, B)$ is said to be nonsplit DS if the induced subgraph $\langle V-\tilde{D}\rangle$ is connected.

The non-split DN $\Upsilon_{\mathrm{ns}}(G)$ of an $m$-PIVFG $G=(V, A, B)$ be minimum cardinality of all non-split DS. Since every non-split DS of an $m$-PIVFG being DS of that $m$-PIVFG, then $\Upsilon(G) \leq \Upsilon_{\mathrm{ns}}(G)$.

Example 10 From (Fig. 1) Example 1, the DS $\{b\}$ be nonsplit. $\langle V-\{b\}\rangle=\{a, c, d, e\}$ being connected implies $\Upsilon_{\mathrm{ns}}(G)=0.9$. In this case $\Upsilon(G)=\Upsilon_{\mathrm{ns}}(G)$. And also if we take $\{a, b, c\}$ as DS then $\langle V-\{a, c\}\rangle=\{b, d, e\}$ is connected \& $\Upsilon_{\mathrm{ns}}(G)=1.85$. That imply, $\Upsilon(G)<\Upsilon_{\mathrm{ns}}(G)$. $\therefore \Upsilon(G) \leq \Upsilon_{\mathrm{ns}}(G)$.

Theorem 9 A non-split DS $\tilde{D}$ of an $m-P I V F G G$ is minimal iff for each vertex $y \in \tilde{D}$ one of the following is satisfied.

i. $\exists x \in V-\tilde{D}$ so that $N(x) \cap \tilde{D}=\{y\}$

ii. $y$ being isolated vertex

iii. $N(x) \cap\langle V-\tilde{D}\rangle=\langle\phi\rangle$.

Proof Let $\tilde{D}$ be minimal DS of an $m$-PIVFG $G=$ $(V, A, B)$. Suppose there exist a vertex $y \in \tilde{D}$ so that $y$ satisfy none of the above three conditions. Then by theo$\operatorname{rem} \tilde{D_{1}}=\tilde{D}-\{v\}$ be a DS. By third condition $\left\langle V-\tilde{D_{1}}\right\rangle$ is connected. This indicate $\tilde{D_{1}}$ is a non-split DS of $G=$ $(V, A, B)$, a contradiction. Hence, our assumption was wrong.

Definition 27 A DS $\tilde{D}$ of $G=(V, A, B)$ is a strong nonsplit DS if the induced subgraph $\langle V-\tilde{D}\rangle$ is complete. The 


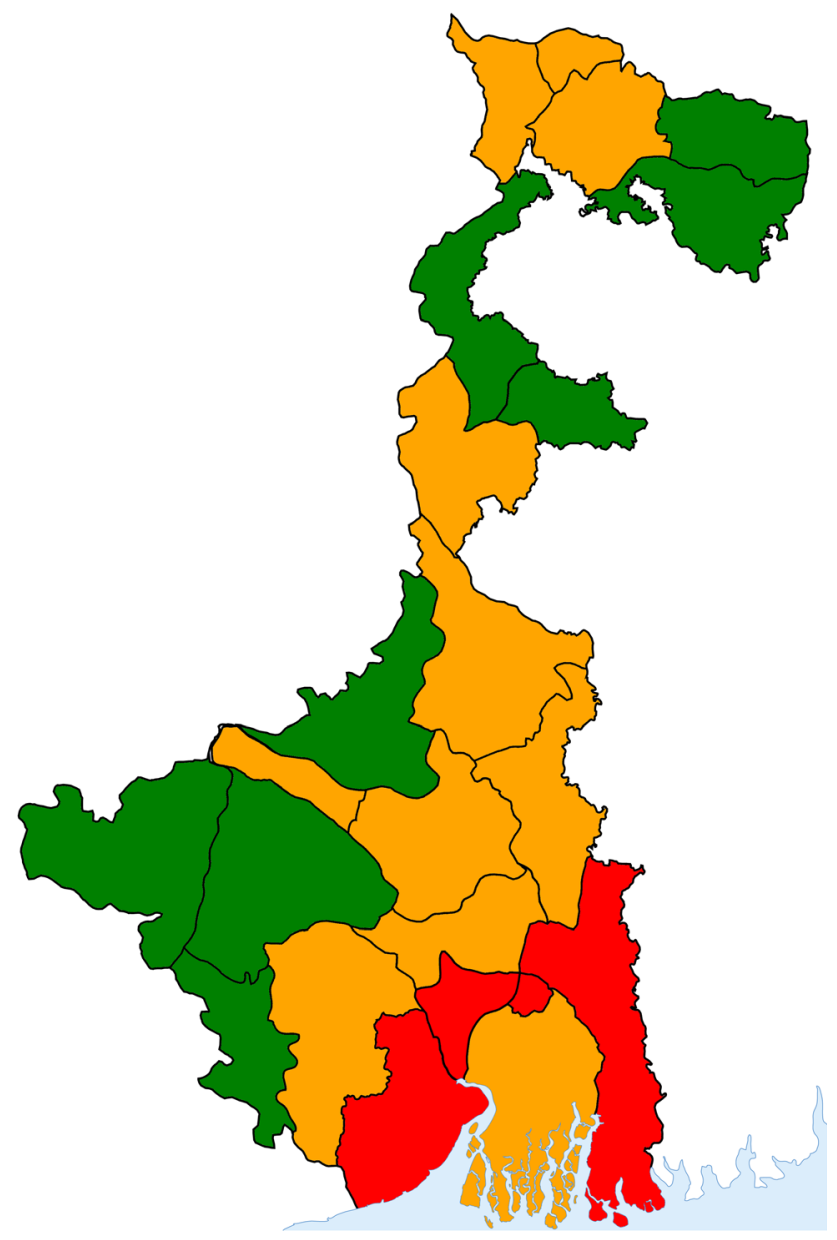

Fig. 5 Zones of West Bengal. Red zones indicate the areas where Covid affected mostly and where lockdown is very necessary. Orange zones mean the areas where Covid affected less and green zones mean Covid free areas (color figure online)

strong non-split DN $\Upsilon_{\text {sns }}$ is the minimum cardinality of strong non-split DS.

Since every strong non-split DS of $G=(V, A, B)$ is a non-split DS, $\Upsilon_{\mathrm{ns}}(G) \leq \Upsilon_{\mathrm{sns}}(G)$. Thus for any $m$-PIVFG, $\Upsilon(G) \leq \Upsilon_{\mathrm{ns}}(G) \leq \Upsilon_{\mathrm{sns}}(G)$.

Definition 28 A DS $\tilde{D}$ of a connected $m$-PIVFG is a cycle non-split DS if the induced subgraph $\langle V-\tilde{D}\rangle$ is a cycle. The cycle non-split DN $\Upsilon_{\text {cns }}$ is the minimum cardinality of cycle non-split DS.
Example 11 Lets consider Example 1 (Fig. 1). Here, if we consider the DS $\{a, c\}$ then the set $\{b, d, e\}$ is a cycle. Henceforth, it is a cycle DS on this 3-PIVFG.

Definition 29 A DS $\tilde{D}$ of a connected $m$-PIVFG is a path non-split DS if the induced subgraph $\langle V-\tilde{D}\rangle$ is a path. The path non-split DN $\Upsilon_{\text {pns }}$ is the minimum cardinality of path non-split DS.

Example 12 Lets consider Example 1 (Fig. 1). Here, if we consider the DSs $\{a, d\},\{b\},\{d, e\},\{c, e\},,\{b, a, e\}\{b, c, d\}$ then the sets are path non-split DS.

Proposition 2 For any non-trivial connected $m$-PIVFG, $\Upsilon_{\text {pns }} \leq \Upsilon_{\text {pns }}$

\subsection{Cototal domination in $m$-PIVFG}

Definition 30 A DS $\tilde{D}$ of an $m$-PIVFG is a cototal DS if the induced subgraph $\langle V-\tilde{D}\rangle$ has no isolated vertices. The cototal DN $\Upsilon_{\text {ct }}$ defined by the minimum cardinality of a cototal DS.

Definition 31 The condition for a DS $\tilde{D}$ of an $m$-PIVFG to be a global non-split DS is $\tilde{D}$ being non-split DS of both $G$ and $\bar{G}$. The global non-split DN $\Upsilon_{\text {gns }}$ means the minimum cardinality of a global non-split DS.

\section{Case study}

Domination in $m$-PIVFG can be applied in a wide variety of several practical problems such as emergency response operations, logistics systems, covering problems, and location-routing problems. Herein, a case study of the domination in $m$-PIVFG is incorporated in order to validate our proposed concept.

\subsection{Disaster response operations}

Disasters are remarkable circumstances that require noteworthy logistical management to distribute essential humanitarian products so as to help and give alleviation to casualties. A productive response assists with diminishing social, financial, and ecological effects. In recent times,

Table 2 Value given to each DC

\begin{tabular}{lllllllllll}
\hline$p(i)$ & $a$ & $b$ & $c$ & $d$ & $e$ & $f$ & $g$ & $h$ & $i$ & $j$ \\
\hline$p(1)$ & {$[0.1,0.4]$} & {$[0.1,0.3]$} & {$[0.2,0.4]$} & {$[0.2,0.5]$} & {$[0.2,0.5]$} & {$[0.4,0.5]$} & {$[0.4,0.6]$} & {$[0.2,0.3]$} & {$[0.2,0.3]$} & {$[0.2,0.4]$} \\
$p(2)$ & {$[0.4,0.7]$} & {$[0.3,0.5]$} & {$[0.6,0.9]$} & {$[0.4,0.7]$} & {$[0.2,0.4]$} & {$[0.1,0.2]$} & {$[0.1,0.3]$} & {$[0.3,0.4]$} & {$[0.1,0.4]$} & {$[0.4,0.6]$} \\
$p(3)$ & {$[0.5,0.7]$} & {$[0.6,0.7]$} & {$[0.2,0.4]$} & {$[0.3,0.4]$} & {$[0.4,0.6]$} & {$[0.3,0.6]$} & {$[0.2,0.5]$} & {$[0.5,0.8]$} & {$[0.4,0.7]$} & {$[0.2,0.7]$} \\
\hline
\end{tabular}




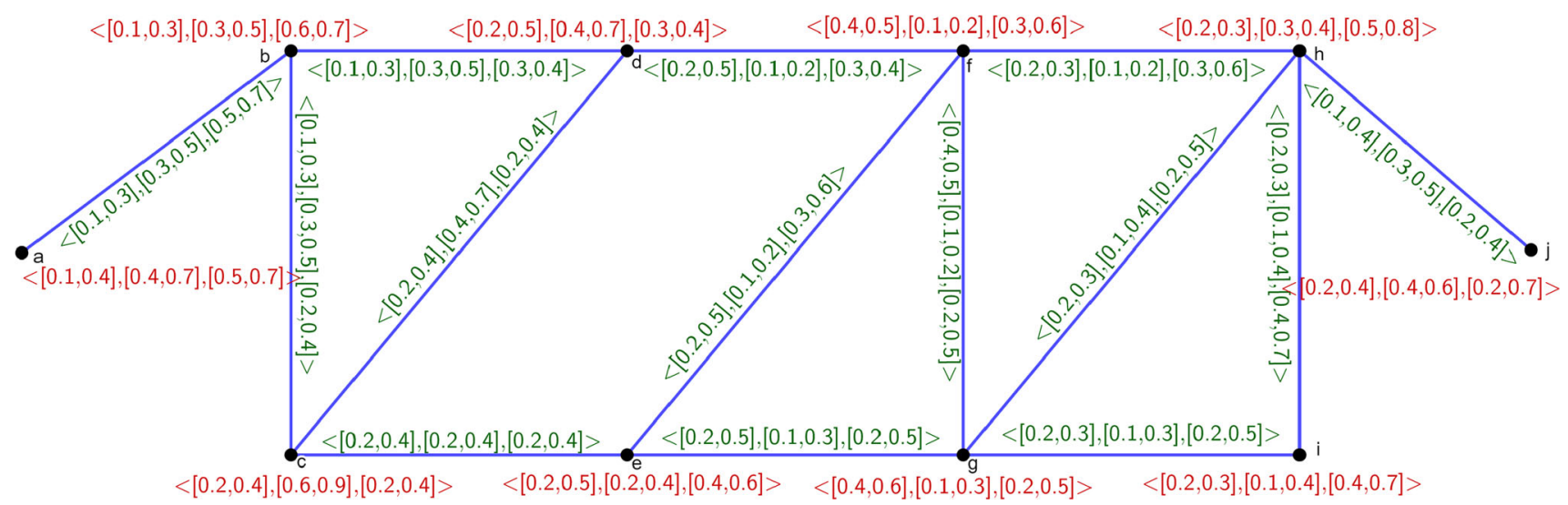

Fig. 6 Pictorial diagram of the application

various natural calamities are cataclysmic the whole World such as Tsunami (2004), Haiti earthquake (2010), earthquake in Nepal (2015), floods in Chennai (2015), Kerala flood (2018), Cyclone Fani in Bangladesh, Sri Lanka, Odisha, East India (2019). Recently, the "COVID-19 pandemic" is an ongoing outbreak which affects more than 3.84 million people in all over World (see https://en.wiki pedia.org/wiki/COVID-19_pandemic, 7 May 2020).

Such type of circumstances can be only handled by providing essential services such as medical aid, feeding operation, sanitation services, distribution of humanitarian items, etc. So as to give these products and ventures to the influenced locales, and to have the option to rapidly react if there should arise an occurrence of crises, an effective and adaptable flexible framework is necessary. In this regard, the covering problem plays a vital role to manage such kind of situations. The affected regions are addressed as the demand points and considered to be covered only if at least a facility is accessible to supply all the necessary services within a given distance. The main aim of the problem is maximizing the coverage demand points with the minimum number of facilities. Now, due to the "COVID-19 pandemic," Department Of Health and Family Welfare, West Bengal, India is announced that the whole state is divided into 04 Red Zones (see Fig. 5, marked by red color) which means the regions are mostly affected. The entry and exit of individuals will be seriously limited. Movement of people will only be allowed for the supply of essential goods and services. Nearly everything will stay shut. In these circumstances, the government wishes to locate some distribution centers (DC) so that each Red Zone will be covered with maximizing the effecting region, minimum logistics cost and conveyance time. In fact, the government aims to set up the minimum number of DC to reduce the opening cost of DC. This scenario can be prevented by a 3PIVFG, $G=(T, R)$, where $T$ is the 3-polar interval-valued fuzzy vertex set of Red Zones (Table 2) and $R$ is the 3- polar interval-valued fuzzy edge set of the relation between zones depending on these criteria such as the effecting region, logistics cost and conveyance time. These criteria are taken as pole, respectively, in the form of some interval taken from $[0,1]$. Here, we have taken 10 number of DC $\{a, b, c, d, e, f, g, h, i, j\}$ in which we have to find minimum number of DC that means minimal domination (Fig. 6).

$$
\begin{aligned}
B= & \left(\frac{a b}{\langle[0.2,0.5],[0.2,0.6],[0.0,0.5]\rangle}, \frac{b c}{\langle[0.1,0.4],[0.2,0.6],[0.0,0.3]\rangle},\right. \\
& \frac{c d}{\langle[0.3,0.5],[0.2,0.8],[0.0,0.2]\rangle}, \frac{c e}{\langle[0.2,0.5],[0.3,0.7],[0.0,0.4]\rangle}, \\
& \frac{d e}{\langle[0.0,0.5],[0.3,0.4],[0.1,0.2]\rangle}, \frac{e f}{\langle[0.1,0.6],[0.4,0.8],[0.2,0.5]\rangle}, \\
& \frac{e g}{\langle[0.2,0.5],[0.3,0.7],[0.0,0.4]\rangle}, \frac{f g}{\langle[0.1,0.4],[0.2,0.5],[0.0,0.2]\rangle}, \\
& \frac{g i}{\langle[0.1,0.6],[0.4,0.8],[0.2,0.5]\rangle}, \frac{g h}{\langle[0.2,0.5],[0.3,0.7],[0.0,0.4]\rangle}, \\
& \frac{h i}{\langle[0.1,0.4],[0.2,0.5],[0.0,0.2]\rangle}, \frac{h i}{\langle[0.1,0.6],[0.4,0.8],[0.2,0.5]\rangle}, \\
& \left.\frac{h j}{\langle[0.2,0.5],[0.3,0.7],[0.0,0.4]\rangle}\right) .
\end{aligned}
$$

Here $p(i)$ indicates the membership value of $i$ th pole. By direct calculation, we find the minimal DS as $\{b, g, h\}$. $\{b, f, h\}$ and $\{b, g, j\}$ are also minimal DS. But finding the DN, we observed that $\{b, f, h\}$ is the minimum DS and the DN $\Upsilon(G)=2.25$. Hence $\mathrm{b}$, $\mathrm{f}$ and $\mathrm{h}$ are, respectively, taken DC so that all the criteria fulfilled by the Govt.

\subsection{Algorithm}

The steps are given for determination of the best potential facilities in our case study, which are as per the following:

Step 1 Firstly, the vertex set $T$ of $V$ and the edge set $R$ of $E$ are chosen in our $m$-PIVFG.

Step 2 Then, the $m$-PIVFG, $G=(T, R)$ is calculated. Now, we find the vertices $x_{j}$ like $p_{i} \circ \mu_{B}^{l}\left(x_{j} x_{k}\right)=p_{i}$ 。 
$\min \left\{\mu_{A}^{l}\left(x_{j}\right), \mu_{A}^{l}\left(x_{k}\right)\right\}, \quad p_{i} \circ \mu_{B}^{u}\left(x_{j} x_{k}\right)=p_{i} \circ \min \left\{\mu_{A}^{u}\left(x_{j}\right)\right.$, $\left.\mu_{A}^{u}\left(x_{k}\right)\right\}$, for $j \neq k$.

Step 3 The set $\tilde{T}_{j} \subseteq Y$ of vertices $x_{j}$ is formulated. If $\cup_{k} x_{k}=Y-\tilde{T}_{j}$ then $\tilde{T}_{j}$ is a DS, else it's not a DS.

Step 4 Afterwards, we repeat the same steps (Steps 2-3) to seek all the DSs $\tilde{T}_{j}$ of $V$.

Step 5 Thus, we get all the DSs $\tilde{T}_{j}$ of $Y$; Stop.

\section{Conclusion and future research direction}

This article has been incorporated the hypothesis of domination with the idea of $m$-PIVFG and explored a few graph-theoretic concepts. Each of these types of ideas has been proposed by suitable examples. We have also investigated the definitions of order, size, cardinality in $\mathrm{m}$ PIVFG. We have explained DN, isolated vertex, total DS, independent set of domination in $m$-PIVFG with suitable examples. Various important results regarding these dominations have been illustrated. Weak and strong domination, split and non-split domination, strong non-split domination, cycle and path non-split domination, co-total and global non-split domination in $m$-PIVFG have been explained with supporting examples and important results. An illustrative example from the field of disaster management problem has been described for demonstrating the real-world example of domination on $m$-PIVFG shows our approach.

We should feature that regarding this investigation, there are distinctive developing regions that we need not demonstrate here as they are outside of our feasible region. In any case, there can be interesting points for future research; for example, one may examine the $m$-PIVFG with various kinds of environments, e.g., Pythagorean graph, $q$ rung fuzzy graph, fuzzy soft graph, competition graph [12]. Such huge numbers of research bearings stay open; thus, researchers can investigate the problem by the various fuzzy graphs. Later on, we will examine different outcomes of $m$-PIVFG and expand them to describe various dynamic issues and covering problems under different uncertainties.

\section{Declarations}

Conflict of interest The authors declare that they have no conflict of interest.

\section{References}

1. Akram M, Dudek WA (2011) Interval-valued fuzzy graphs. Comput Math Appl 61(2):289-299
2. Akram M, Waseem N (2016) Certain metrics in $m$-polar fuzzy graphs. New Math Nat Comput 12(02):135-155

3. Akram M, Waseem N (2017) Novel decision making method based on domination in $m$-polar fuzzy graphs. Commun Korean Math Soc 32(4):1077-1097

4. Akram M, Waseem N, Davvaz B (2017) Certain types of domination in $m$-polar fuzzy graphs. J Mult Valued Logic Soft Comput 29(6)

5. Bera S, Pal M (2020) Certain types of $m$-polar interval-valued fuzzy graph. J Intell Fuzzy Syst 39(3):3137-3150. https://doi.org/ 10.3233/JIFS-191587

6. Bera S, Pal M (2020) On m-polar interval-valued fuzzy graph and its application. Fuzzy Inf Eng (Preprint). https://doi.org/10.1080/ 16168658.2020.1785993

7. Berge C (1973) Graphs and hypergraphs. North-Holland, Amsterdam

8. Bhattacharya P (1987) Some remarks on fuzzy graphs. Pattern Recogn Lett 6(5):297-302

9. Chen J, Li S, Ma S, Wang X (2014) $m$-Polar fuzzy sets: an extension of bipolar fuzzy sets. Sci World J

10. Cockayne EJ, Hedetniemi ST (1977) Towards a theory of domination in graphs. Networks 7(3):247-261

11. Cockayne EJ, Dawes R, Hedetniemi ST (1980) Total domination in graphs. Networks 10(3):211-219

12. Das S, Ghorai G (2020) Analysis of the effect of medicines over bacteria based on competition graphs with picture fuzzy environment. Comput Appl Math 39(3):1-21

13. Gani AN, Ahamed MB (2007) Strong and weak domination in fuzzy graphs. East Asian Math J 23(1):1-8

14. Ghorai G, Pal M (2015) On some operations and density of $m$ polar fuzzy graphs. Pac Sci Rev A Nat Sci Eng 17(1):14-22

15. Ghorai G, Pal M (2016) Some properties of $m$-polar fuzzy graphs. Pac Sci Rev A Nat Sci Eng 18(1):38-46

16. Ghorai G, Pal M (2017) On degrees of $m$-polar fuzzy graphs with application. J Uncertain Syst 11(4):294-305

17. Ghorai G, Pal M (2018) Applications of bipolar fuzzy sets in interval graphs. TWMS J Appl Eng Math 8(2):411

18. Hongmei J, Lianhua W (2009) Interval-valued fuzzy subsemigroups and subgroups associated by interval-valued fuzzy graphs. In: 2009 WRI global congress on intelligent systems, vol 1, pp 484-487. IEEE

19. Jana C, Pal M, Jq Wang (2020) Bipolar fuzzy Dombi prioritized aggregation operators in multiple attribute decision making. Soft Comput 24(5):3631-3646

20. Kaufmann A (1975) Introduction to the theory of fuzzy subsets, vol 2. Academic Press

21. Mahapatra R, Samanta S, Allahviranloo T, Pal M (2019) Radio fuzzy graphs and assignment of frequency in radio stations. Comput Appl Math 38(3):117

22. Merouane HB, Chellali M (2015) On secure domination in graphs. Inf Process Lett 115(10):786-790

23. Mordeson JN, Chang-Shyh P (1994) Operations on fuzzy graphs. Inf Sci 79(3-4):159-170

24. Nagoorgani A, Chandrasekaran V (2006) Domination in fuzzy graph. Adv Fuzzy Sets Syst 21(1):17-26

25. Nagoorgani A, Malarvizhi J (2008) Isomorphism on fuzzy graphs. Int J Comput Math Sci 2(4):190-196

26. Nagoorgani A, Radha K (2008) On regular fuzzy graphs. J Phys Sci $12: 33-40$

27. Ore O (1962) Theory of graphs, volume 38 of. In: Am. Math. Soc. Colloquium Publications

28. Parvathi R, Thamizhendhi G (2010) Domination in intuitionistic fuzzy graphs. Notes Intuitionistic Fuzzy Sets 16(2):39-49

29. Pramanik T, Samanta S, Pal M (2016) Interval-valued fuzzy planar graphs. Int J Mach Learn Cybern 7(4):653-664 
30. Rashmanlou H, Pal M, Borzooei RA, Mofidnakhaei F, Sarkar B (2018) Product of interval-valued fuzzy graphs and degree. J Intell Fuzzy Syst 35(6):6443-6451

31. Rosenfeld A (1975) Fuzzy graphs. In: Fuzzy sets and their applications to cognitive and decision processes. Elsevier, pp 77-95

32. Samanta S, Pal M (2011) Fuzzy tolerance graphs. Int J Latest Trends Math 1(2):57-67

33. Samanta S, Pal M (2013) Fuzzy $k$-competition graphs and $p$ competition fuzzy graphs. Fuzzy Inf Eng 5(2):191-204

34. Shannon A, Atanassov K (2006) On a generalization of intuitionistic fuzzy graphs. NIFS 12(1):24-29

35. Somasundaram A (2005) Domination in products of fuzzy graphs. Int $\mathbf{J}$ Uncertain Fuzziness Knowl Based Syst 13(2):195-204

36. Somasundaram A, Somasundaram S (1998) Domination in fuzzy graphs-i. Pattern Recogn Lett 19(9):787-791

37. Sunitha M (2001) Studies on fuzzy graphs. Depart. of Mathematics, Cochin university of Science and Technology, Cochin, India
38. Sunitha M, Vijayakumar A (2002) Complement of a fuzzy graph. Indian J Pure Appl Math 33(9):1451-1464

39. Talebi A, Rashmanlou H (2013) Isomorphism on interval-valued fuzzy graphs. Ann Fuzzy Math Inform 6(1):47-58

40. Zadeh LA (1965) Fuzzy sets. Inf Control 8(3):338-353

41. Zhang WR (1994) Bipolar fuzzy sets and relations: a computational framework for cognitive modeling and multiagent decision analysis. In: NAFIPS/IFIS/NASA'94. Proceedings of the first international joint conference of the North American fuzzy information processing society biannual conference. The industrial fuzzy control and intelligence. IEEE, pp 305-309

42. Zhang WR (1998) Bipolar fuzzy sets. In: 1998 IEEE international conference on fuzzy systems proceedings. IEEE world congress on computational intelligence (Cat. No. 98CH36228), vol 1. IEEE, pp 835-840

Publisher's Note Springer Nature remains neutral with regard to jurisdictional claims in published maps and institutional affiliations. 